OPEN ACCESS

Edited by:

Alessandro Poggi,

San Martino Hospital (IRCCS), Italy

Reviewed by:

Vita Golubovskaya,

University of Oklahoma Health

Sciences Center, United States

Zahid Pranjol,

University of Sussex, United Kingdom

*Correspondence:

Feng Luo

luofeng66666@sina.com

Jun $\mathrm{He}$

he-001jun@163.com

${ }^{\dagger}$ These authors have contributed equally to this work

Specialty section:

This article was submitted to

Cancer Immunity

and Immunotherapy,

a section of the journal

Frontiers in Immunology

Received: 27 October 2021 Accepted: 01 February 2022

Published: 23 February 2022

Citation:

Hu H, Chen Y, Tan S, Wu S, Huang Y,

Fu S, Luo F and He J (2022) The Research Progress of Antiangiogenic

Therapy, Immune Therapy and Tumor Microenvironment.

Front. Immunol. 13:802846. doi: 10.3389/fimmu.2022.802846

\section{The Research Progress of Antiangiogenic Therapy, Immune Therapy and Tumor Microenvironment}

\author{
Haoyue $\mathrm{Hu}^{1,2 \dagger}$, Yue $\mathrm{Chen}^{3 \dagger}$, Songtao $\mathrm{Tan}^{1 \dagger}$, Silin $\mathrm{Wu}^{4}$, Yan Huang ${ }^{1}$, Shengya $\mathrm{Fu}^{1,5}$, \\ Feng $\mathrm{LuO}^{1 *}$ and Jun $\mathrm{He}^{6 *}$ \\ ${ }^{1}$ Lung Cancer Center, Cancer Center, State Key Laboratory of Biotherapy, West China Hospital of Sichuan University, \\ Chengdu, China, ${ }^{2}$ Department of Medical Oncology, Sichuan Cancer Hospital and Institute, Sichuan Cancer Center, \\ Medicine School of University of Electronic Science and Technology, Chengdu, China, ${ }^{3}$ Department of Pathology, Beijing \\ Shijitan Hospital, Capital Medical University, Beijing, China, ${ }^{4}$ School of Pharmacy, Southwest Medical University, \\ Luzhou, China, ${ }^{5}$ Second Department of Oncology, Sichuan Friendship Hospital, Chengdu, China, ${ }^{6}$ Department of Oncology, \\ The Third Hospital of Mianyang (Sichuan Mental Health Center), Mianyang, China
}

Anti-angiogenesis therapy, a promising strategy against cancer progression, is limited by drug-resistance, which could be attributed to changes within the tumor microenvironment. Studies have increasingly shown that combining anti-angiogenesis drugs with immunotherapy synergistically inhibits tumor growth and progression. Combination of anti-angiogenesis therapy and immunotherapy are well-established therapeutic options among solid tumors, such as non-small cell lung cancer, hepatic cell carcinoma, and renal cell carcinoma. However, this combination has achieved an unsatisfactory effect among some tumors, such as breast cancer, glioblastoma, and pancreatic ductal adenocarcinoma. Therefore, resistance to anti-angiogenesis agents, as well as a lack of biomarkers, remains a challenge. In this review, the current antiangiogenesis therapies and corresponding drug-resistance, the relationship between tumor microenvironment and immunotherapy, and the latest progress on the combination of both therapeutic modalities are discussed. The aim of this review is to discuss whether the combination of anti-angiogenesis therapy and immunotherapy can exert synergistic antitumor effects, which can provide a basis to exploring new targets and developing more advanced strategies.

Keywords: antiangiogenic therapy, immune therapy, tumor microenvironment, cancer biology, progress

\section{CURRENT STATUS OF ANTI-TUMOR ANGIOGENESIS THERAPY}

In 1971, Folkman hypothesized that "neovascularization is critical for tumors growth". Since then, anti-tumor angiogenesis therapy has gained considerable attention, and is currently one of the most effective methods to treat cancer. Tumor blood vessels are fundamental for tumor growth and metastasis. Tumor angiogenesis is regulated by a variety of cytokines. The vascular endothelial growth factor (VEGF) family regulates the growth of blood vessels. In mammals, there are five 
isoforms within the VEGF family, including VEGF-A, VEGF-B, VEGF-C, VEGF-D, and placental growth factor (PGF). These proteins correspond to three different tyrosine kinase receptors, known as VEGFR1, VEGFR2, and VEGFR3. Studies have demonstrated that VEGF is highly expressed in different types of tumors, including vascular endothelial growth factor receptor (VEGFR1), VEGFR2, and VEGFR3. VEGFR2 plays a significant role in angiogenesis. VEGFR2 can activate the MAPK and PI3K signal pathways, which, in turn, activates the downstream ERK1/ 2 or mTOR ligand, leading to tumor growth and angiogenesis (Figure 1). Therefore, most anti-angiogenic drugs target the VEGF signaling system (ligands, receptors, and intracellular downstream pathways). Over the past 20 years, dozens of antiangiogenic drugs have been granted approval for treatment of multiple cancer types. One meta-analysis of randomized phase II/III trials (1) showed that, compared to platinum-based chemotherapy alone, the combination of bevacizumab and chemotherapy significantly prolonged survival of previously untreated patients with advanced non-small-cell lung cancer (NSCLC) $(\mathrm{HR}=0.90,95 \% \mathrm{CI}: 0.81-0.99, P=20)$. In the phase III LUME - Lung1 trial, the combination of docetaxel and nintedanib increased the median overall survival (OS) of lung adenocarcinoma patients, who had relapsed within 9 months of first-line chemotherapy, from 7.9 months to 10.9 months $(\mathrm{HR}=$ 0.75, 95\% CI: $0.60-0.92, P=0.0073$ ) (2). In the second cohort, the combination treatment also provided a survival benefit (median OS of docetaxel + nintedanib group $v s$ control group $=12.6$ months $v s 10.3$ months; $\mathrm{HR}=0.83$, CI:0.70-0.99, $P=0$. 0359). The phase III REVEL trial (3) compared the therapeutic effect of docetaxel alone, as well as in combination with ramucirumab, in advanced NSCLC patients recalcitrant to platinum-based dual-drug chemotherapy. The median survival duration of the combination treatment group was 10.5 months, while the docetaxel group was 9.9 months $(\mathrm{HR}=0.86,95 \% \mathrm{CI}$ : $0.75-0.98, P=0.023)$. Subsequently, docetaxel and ramucirumab were granted approval by both EMA and FDA for treating metastatic NSCLC.

However, anti-tumor angiogenesis therapy has shown limited efficacy, with survival benefits ranging from only a few weeks to months. On the other hand, other studies have reported tumor progression (4) during anti-angiogenesis treatment. For example, Kindler et al. (5) compared the therapeutic effect of gemcitabine alone and combined with bevacizumab on advanced pancreatic cancer patients, and found that the combination therapy did not improve OS. Similarly, a significant recurrence rate was seen among glioma patients treated with bevacizumab (6). Studies on xenograft models of melanoma or breast cancer (7) showed that sunitinib, in fact, increased metastasis and shortened the survival of the tumor-bearing mice. Recently, the China Food and Drug Administration (CFDA) approved the single agent anlotinib as a third-line treatment for patients with advanced NSCLC. Compared to placebo, anlotinib has been demonstrated to improve both progression-free survival (PFS) and overall survival (OS) in a phase III trial among patients with advanced non-small-cell lung cancer (NSCLC), despite progression of cancer after two lines of prior treatments.

\section{THE RESISTANCE TO ANTIANGIOGENIC THERAPY}

Among normal healthy human beings, angiogenic balance exists due to regulation of the vascular endothelial growth factors (VEGFs) - angiostatin and the angiogenic molecules. However, in tumors, this balance becomes converted to pro-angiogenesis. VEGFs, which play pivotal roles in wound healing and angiogenesis, consist of five members, VEGF A-E. VEGF works by binding to the vascular endothelial growth factor receptor (VEGFR), which is comprised of three tyrosine kinases. VEGA-A is up-regulated in most solid tumors, including breast cancer and

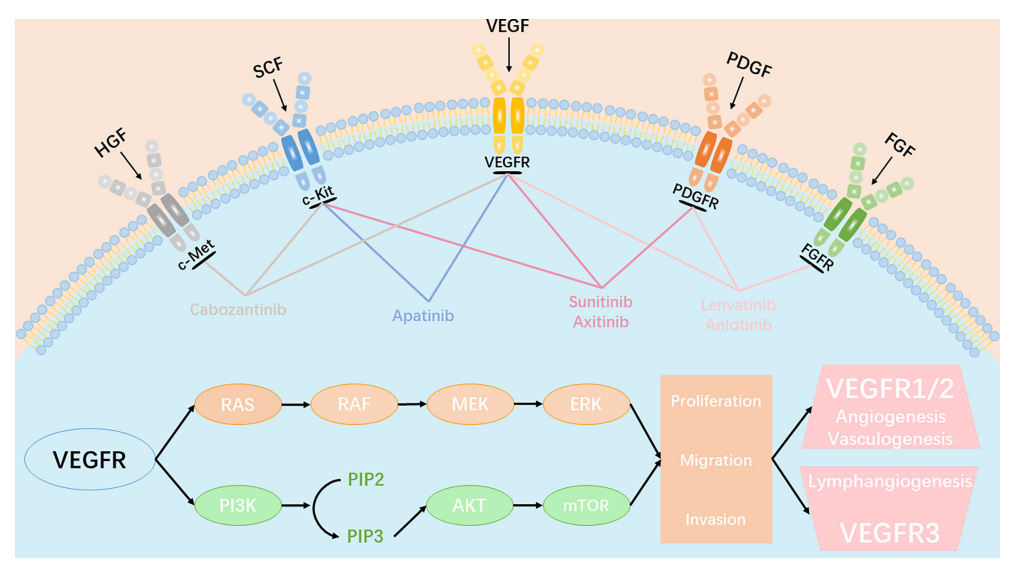

FIGURE 1 | Angiogenic signaling pathway and key anti-angiogenic targets in tumor angiogenesis. VEGFR、PDGFR、FGFR、C-kit、C-Met involved in the key molecular signal events(RAS-RAF-MEK-ERK signaling pathway and PI3K-AKT-mTOR signaling pathway) which plays a significant role in tumor proliferation, migration and invasion. All approved angiogenic tyrosine kinase inhibitors (TKIs) can target multiple receptors simultaneously and inhibit the transduction of downstream signaling. 
lung cancer, which makes it a significant target of antiangiogenesis drugs (8). The first FDA-approved antiangiogenesis treatment was kinase inhibitors that targeted the VEGFR. Subsequently, antibodies against VEGF-Trap and VEGFR2 have been approved (9). Hepatocyte growth factor (HGF) promotes cell survival, enhances cell invasion ability, and facilitates epithelial-mesenchymal transition by activating the mesenchymal-epithelial transformation factor (c-MET) signaling pathway in endothelial cells. To date, researchers have discovered that c-MET and HGF are overexpressed in a number of tumors, which has led to abnormal gene amplification, activation of transcription or hypoxia microenvironment. Aberrant HGF/Met signaling is associated with poor prognosis in several tumor types. HGF/Met signaling stimulates several pathways, including MAPK signal pathway, which we mentioned above. PI3K signal pathway and $\mathrm{Wnt} / \beta$ catenin pathways play significant roles in cell proliferation, survival, and angiogenesis. However, clinical efficacy of VEGFtargeted drugs has vital limitations. Although phase 3 trials have demonstrated that use of anti-angiogenic agents leads to significant improvements in overall survival (OS) for several cancers, such as advanced-stage CRC, RCC, and HCC, it is also associated with a failure to improve OS in other cancers, such as breast cancer, glioblastoma, pancreatic ductal adenocarcinoma (PDAC) and prostate cancer (10-13). Carvalho B et al. discovered that, in glioblastoma, c-Met overexpression is associated with a time-to-progression (TTP) after bevacizumab of 3 months (95\% CI, 1.5-4.5), compared with a TTP of 7 months (95\% CI, 4.6-9.4) among patients with low or no expression of $c$-Met $(p=0.05)$. VEGFR2 expression was associated with a TTP after bevacizumab treatment at 3 months (95\% CI, 1.8-4.2) compared with a TTP at 7 months (95\% CI, 5.7-8.3) among patients with no expression of VEGFR2 $(p=0.009)$. Concomitant $c$-Met/VEGFR2 overexpression was found to be associated with worse overall survival (13 months) compared to concomitant c-Met/VEGFR2 negative expression (19 months; $p=0.025$ ). Their data indicates that $c$-Met and VEGFR2 overexpression play a significant role in the development of glioblastoma early resistance and may predict poorer responses to anti-angiogenic therapies (13).

Either anti-VEGFs or anti-VEGFRs or other nonspecific tyrosine kinase inhibitors ultimately shut down tumor blood supply and drive tumor necrosis. Necrosis usually occurs in the central part of the tumor, and the surrounding tumor cells remain alive as they are benefited by nutrition delivered by nearby normal blood vessels. As a result, most vascular disruptor therapy does not completely prevent tumor growth. This may be one of the reasons why antiangiogenic therapy improves therapeutic outcomes, while beneficial effects remain short (9). In addition, primary or acquired resistance contributes more to failure of anti-angiogenesis treatment. Tina Cascone used mouse-and human-specific profiling of human NSCLC xenografts in mice in order to investigate stromal and tumor cell changes that occur in tumors that acquire resistance to antiangiogenesis treatment. Researchers found that changes in gene expression, particularly changes in expression of angiogenesis- related genes, occurred predominantly in stromal cells, but not in tumor cells. The observation reinforces the notion that tumor stroma may play an important and potentially dominant role, in at least some circumstances - in VEGF inhibitor resistance (14). Furthermore, extrinsic mechanisms have also been shown to be involved in resistance to antiangiogenic therapy, including changes in the tumor microenvironment (TME), the presence of cancer stem cells (CSCs), and tumor immunosuppression, which significantly limits their clinical value $(15,16)$.

\section{TUMOR MICROENVIRONMENT AND ANTIANGIOGENIC THERAPY}

The tumor microenvironment (TME) is composed of immune cells, stromal cells, extracellular matrix (ECM), blood vessels, tumor cells, lymphatic vessels and CSCs. The constant changes that occur in the various components of the TME result in its complexity and heterogeneity. TME is associated with multiple processes, including proliferation, angiogenesis, apoptosis, and immune surveillance. The stromal cells, particularly cancerassociated fibroblasts (CAFs), can promote tumor cell survival mainly by recruiting immune cells into the TME, and promote invasion by constructing a hypoxic environment. Tumor-driven hypoxia, increased inflammation, or MMPs overexpression in the TME induces alterations in the ECM, following the tumor biological behavior of evading apoptosis, elevating invasion and metastasis $(17,18)$. In addition, ECM components can regulate the cancer-immunity cycle. The above TME changes cause tumor progression and drug resistance (19). As tumors generally tend to be hypoxic, prolonged use of antiangiogenesis drugs can often aggravate hypoxia $(20,21)$. As previously reported, the upregulation of hypoxic inducible factor 1a (HIF-1a) is also responsible for heterogeneity of breast cancer, lung cancer, cervical carcinoma, and gliomas (22-24). Hypoxiainduced upregulation of HIF-1a can mediate tumor cell dedifferentiation into CSCs, which is a primary mechanism that underlies resistance to anti-angiogenesis therapy $(25,26)$. In addition, HIF-1a upregulates the expression of nuclear factor-k $\beta$ and leads to increased recruitment of monocytes and tumorassociated macrophages (TAMs), including polarization of the M2 phenotype TAMs, which promotes recurrence and metastasis (27). The immune system can have a dual effect in cancer biology, including pro-tumorigenic and anti-tumorigenic effect. The immune surveillance system identifies, kills, and removes tumor cells from the body. NK cells, CD8+ cytotoxic $\mathrm{T}$ cells and the major histocompatibility complex (MHC) class 1 molecules are known to play major roles in the function of immunosurveillance. Unsurprisingly, the host immune system is often disrupted and creates an immune imbalance among cancer patients. Tumor cells can camouflage themselves in order to hide from immune cells, thus avoiding being discovered. Numerous cellular and molecular mechanisms have been shown to be responsible for tumor evasion $(28,29)$. Immunosuppressive cells, such as T-regs, TAMs, and MDSCs frequently accumulate within the TME, which is associated with an 
unfavorable prognosis. When there are a large number of immune cells in tumor tissues, such as T-regs, MDSCs, TAMs, and DCs, they can promote an immunosuppressive microenvironment and participate in immune escape. Antiangiogenic therapy for VEGF or VEGF receptor-2 (VEGFR-2) can increase the transport of $\mathrm{T}$ cells to the tumor, thereby reducing immunosuppressive cytokines and T-regs, helping overcome resistance to the checkpoint inhibitors' medicinal properties (30) (Figure 2). Furthermore, tumor cells also secrete immunosuppressive cytokines, such as IL-10 and TGF$\beta$, which are known to inhibit perforin and production by CD8+ $\mathrm{T}$ cells and inactivate cytotoxicity of NK cells (31-33).

Additionally, primary drug resistance due to a lack of tumorinfiltrating lymphocytes in the tumor should not be ignored. In addition to the VEGF pathway, angiogenesis can be induced by the angiopoietin (Ang1-2)/Tie-2 pathway. Studies have shown that patients that receive immunotherapy with higher Ang-2 expression tend to have poorer clinical outcomes. This suggests that the Ang-2 pathway is another cause of immunotherapy resistance $(34,35)$.

\section{THE EFFICACY OF ANTI-CANCER IMMUNOTHERAPY}

Under normal circumstances, the immune system can recognize and eliminate tumor cells within the tumor microenvironment. However, in order to survive, tumor cells can adopt different strategies in order to suppress the immune system and protect itself from CD8+ T cells. Immunotherapy has heralded a new era of oncotherapy and aims to either directly eliminate cancer cells or activate the host immune response. It is mediated through anticancer cell vaccines and antibodies, cytokines, adoptive immune cell transfer and immune checkpoint blockers (ICBs). Tumor immunotherapy is a treatment that restores the body's normal anti-tumor immune response by restarting and maintaining the tumor-immune cycle, thereby controlling and eliminating tumor cells. Tumor immunotherapy includes monoclonal antibody immune checkpoint inhibitors (36), therapeutic antibodies, cancer vaccines, cell therapy and small molecule inhibitors. In recent years, cancer immunotherapy has continued to progress. At present, this treatment method has shown strong anti-tumor activity in the treatment of solid tumors such as melanoma, NSCLC, kidney cancer, and prostate cancer. Furthermore, immunotherapy drugs have been approved by the US FDA (Food and Drug Administration) for clinical application (37). Moreover, increasing evidence has shown that overexpression of vascular growth factors can activate immunosuppressive cells directly and suppress immune effector cells to alter the immunosuppressive microenvironment.

\section{COMBINATION OF IMMUNOTHERAPY AND ANTI-TUMOR ANGIOGENESIS}

The relationship between angiogenesis and immune therapy is a complicated interplay. Anti-angiogenic agents can stimulate the immune system and improve the immunosuppressive environment, while immunotherapy can also have antiangiogenesis effects. Therefore, there is a synergistic relationship between the two treatment methods $(38,39)$. Tumor cells can evade $\mathrm{T}$ cell-mediated killing by up-regulating the interaction of PD-L1 with the inhibitory receptor PD-1, which is expressed on tumor-infiltrating T-cells. Tumor cells can evade $\mathrm{T}$ cell-mediated killing by upregulating the interaction of ligands (such as PD-L1) with the inhibitory receptor PD-1, CTLA-4, and LAG-3, which are expressed on tumor-infiltrating T-cells (40). It is inevitable that patients will develop resistance to immune checkpoint inhibitors

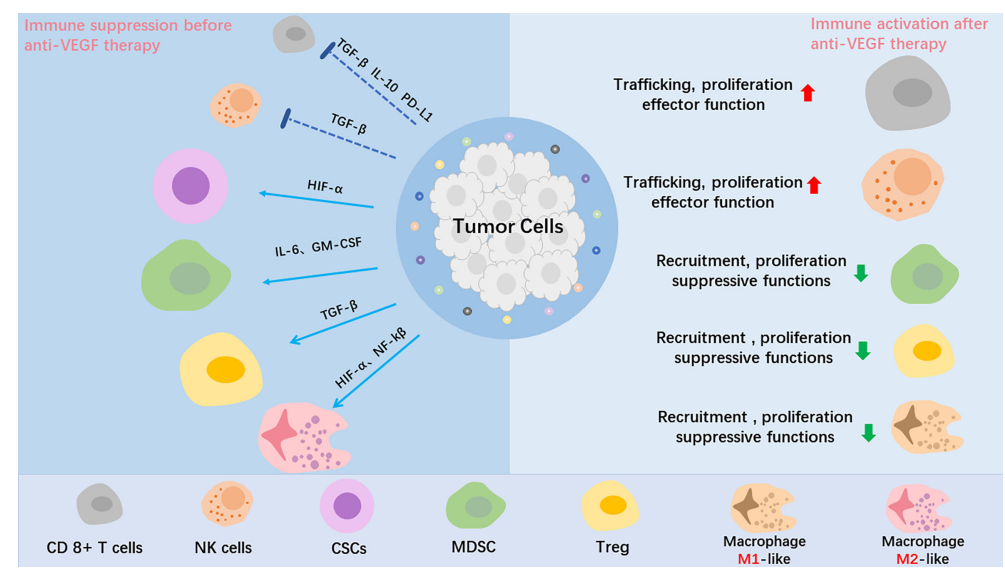

FIGURE 2 | The role of anti-VEGF treatment in the tumor microenvironment (TME). Tumor angiogenesis creates a hypoxic tumor microenvironment, which impedes T-effector cells、NK cells and DC cells infiltration into tumor, mediates tumor cell de-differentiation into CSCs, promotes proliferation of immunosuppressive cells, including Tregs and MDSCs, and polarizes TAMs to the immune inhibitory M2-like phenotype. After anti-VEGF treatment, the anti-tumor factors increase, and the pro-tumor factors are decreased. In summary, anti-VEGF treatment alleviate the immunosuppressive tumor microenvironment and improve cancer immunotherapy. 
due to a lack of PD-L1 and the inhibitory effect in the TME. Facing a complex TME, the key strategy is to inhibit angiogenesis, and an effective immune response (41).

\section{Anti-Angiogenesis Produces Vessel Normalization and Stimulates Immune Responses}

The formation of blood vessels in malignant tumors is largely caused by hypoxia and the excessive secretion of VEGF. Antiangiogenic therapy for VEGF or VEGFR-2 can increase the transport of $\mathrm{T}$ cells to the tumor, thereby reducing immunosuppressive cytokines and regulatory $\mathrm{T}$ cells, which may help overcome resistance to checkpoint inhibitors' medicinal properties (30). A case study of immune checkpoint inhibitors combined with anti-angiogenic drugs in the treatment of metastatic renal cell carcinoma demonstrated that antigenspecific $\mathrm{T}$ cell migration and expression of MHC-1 and PD-L1 were increased. Furthermore, anti-tumor activity was enhanced with less toxicity (42). Tumor blood vessels were found to be highly abnormal, with tumor vessels showing structural abnormalities, leading to hypoxia, acidity, and a high interstitial fluid pressure microenvironment. These microenvironmental abnormalities can affect immune cell proliferation, infiltration, survival, and function (43). Myeloid-derived suppressor cells (MDSCs) are one of the most important stromal cells of the TME, and protect tumor cells from the host immune system by suppressing T-cell function (44). There is evidence to support the hypothesis that anti-angiogenic therapy and immunotherapy act synergistically (45). GM-CSF, a potent cytokine promoting the differentiation of myeloid cells such as dendritic cells, macrophages and granulocytes, which elicits antitumor immunity by enhance tumor antigen presentation to $\mathrm{T}$ cells, has been proven to be effective across numerous clinical trials (46-50).

Another study has reported that binding of tumor-derived VEGF to VEGFR on CD34+ bone marrow progenitor cells reduces the differentiation of these cells into dendritic cells, thus limiting the efficacy of GM-CSF-related immunotherapy (51). And Sylvie et al. also included that human GFs in vitro actively inhibit the differentiation of monocyte-derived dendritic cells through the secretion of IL- 6 and VEGF, limiting the immunotherapy of GM-CSF (52). Furthermore, it also promotes proliferation of immunosuppressive cells, such as Tregs and MDSCs, and inhibits DC maturation, and restricts the development of $\mathrm{T}$ lymphocytes from the lymphoid progenitors $(30,53-55)$. A study (56) on three different NSCLC animal models demonstrated that combining adoptive transfer of cytokine-induced killer (CIK) cells with recombinant human endostatin significantly inhibited angiogenesis and tumor growth, whereas neither was effective when used alone.

\section{Blocking VEGF Induced Immune Checkpoint Expression}

Lydia Meder et al. conducted an experiment on five groups on the combined use of vehicle, IgG, VEGF inhibitor, PD-L1 inhibitor, VEGF inhibitor, and PD-L1 inhibitor in a mouse model of small cell lung cancer. The results indicate that treatment with VEGF, compared to any other treatment methods, the combination of inhibitor and PD-L1 inhibitor greatly improved PFS and OS in mice (57). S. Yasuda et al. reported that in a mouse model of colon cancer, the combined use of PD-1 inhibitors and VEGFR2 inhibitors demonstrated no obvious toxicity. Compared to the control group, the experimental group drugs were found to better inhibit tumor growth. The author believes that the combined use of inhibitors can produce a synergistic anti-tumor effect in the body through a variety of mechanisms, including anti-VEGFR2 therapy resulted in a significant decrease of tumor micro vessels as well as reducing tumor vasculature and anti-PD-1 mAb treatment enhanced the infiltration of $\mathrm{T}$ cells into tumors. And that the two drugs are not mutually exclusive (58). Since immunotherapy has been proved to be effective against CSCs and the immunosuppressive TME, it is reasonable to surmise that a combination of anti-angiogenesis and immunotherapies would have a synergistic effect against recalcitrant tumors. Indeed, studies have shown that (38) targeting the angiogenic factor VEGF, as well as its receptors, stimulates onco-immunity, since VEGF is known to be involved in the immune escape of tumors. The VEGF signaling pathway can abrogate the effects of antitumor therapy via various mechanisms. Usually, is LFA1 that can interact on ICAM1. LFA1 is expressed on lymphocytes and it is a crucial for $\mathrm{T}$ cell entry into mammalian lymph nodes and tissues while ICAM1 on tumor target cells or endothelial cells (59). Previous study showed that clustering of ICAM-1 was indeed prevented by VEGF and a reduced induction of ICAM-1 and VCAM-1 mRNA transcripts by TNF in the presence of VEGF (60). VEGF can also block T-cell activation and induce apoptosis via the Fas/FasL pathway (61). Therefore, blocking VEGF and its receptor can help stimulate immune responses and improve immunotherapy outcomes. Studies on tumor-bearing mouse models have demonstrated that $(62,63)$ multi-targeted antiangiogenic tyrosine kinase inhibitors (TKIs) increased tumor infiltration of CD8+ and CD4+ T cells by downregulating PD-1 expression, and decreased the number and activity of Tregs and MDSCs (64). Similarly, sunitinib inhibited the expansion of Tregs and MDSCs in patients with renal cell carcinoma (30, 63, 65). The VEGFR2-targeting TKI cabozantinib was also associated with a reduction in the number of Tregs and MDSCs, and simultaneously promoted tumor infiltration of CD4+ and CD8+ T lymphocytes, both alone and in combination with the anti-cancer vaccine $\mathrm{MVA} / \mathrm{rF}-\mathrm{CEA} /$ TRICOM (65). VEGFR1/R2 and soluble chimeric VEGF receptor, can bind to VEGF with high affinity and efficiently play an anti-angiogenic therapy function. In animal experiments, the combination of sVEGFR1/R2 therapy and GM-CSFsecreting tumor cell immunotherapy can remarkably prolong the survival of tumor model mice $(66,67)$. Similarly, the combination of cabozantinib and MVA/rF-CEA/TRICOM was found to significantly inhibit growth of MC38-CEA tumors in a mouse model (65). In a mouse model of colon cancer, anti-PD-1 monoclonal antibodies and VEGFR2 resulted in significantly greater tumor inhibition compared to either monotherapy (58). 


\section{CLINICAL USE OF ANTIANGIOGENIC DRUGS AND ICBS AGAINST TUMORS}

The relationship between angiogenesis and immune therapy has been suggested to be a complicated interplay (68). Antiangiogenic agents are known to stimulate the immune system and improve the immune suppression environment (69). Furthermore, immunotherapy can also cause anti-angiogenesis effects, and there is a synergistic relationship between the two treatment methods (39). Tumor cells can evade T cell-mediated killing by up-regulating the interaction of PD-L1 with the inhibitory receptor PD-1 that is expressed on tumorinfiltrating $\mathrm{T}$ cells. Thus, it is inevitable that patients develop resistance to immune checkpoint inhibitors due to a lack of PDL1 and the inhibitory effect in the tumor microenvironment. The therapy should inhibit angiogenesis, on the other hand trigger anti-tumor immunity (41). The formation of blood vessels in malignant tumors is mainly caused by hypoxia and excessive secretion of vascular endothelial growth factor (VEGF). Recently, an accumulating number of clinical trials have been conducted to explore the efficacy of the combination of anti-angiogenesis and immunotherapy (Table 1).

\section{Clinical Trials on NSCLC}

In a phase 3 clinical trial IMpower150 (NCT02366143), 1202 patients with metastatic non-squamous NSCLC (ns-NSCLC) were treated with a combination of Atezolizumab to Bevacizumabbased chemotherapy, including three groups: (1) Atezolizumab, Carboplatin, and Paclitaxel (ACP); (2) Atezolizumab, Bevacizumab, Carboplatin and Paclitaxel (ABCP); (3) Bevacizumab, Carboplatin and Paclitaxel (BCP). The results demonstrated that the $\mathrm{ABCP}$ group had significantly improved PFS and OS, with an average of 8.3 and 19.2 months, respectively, both of which were better than the control group (70). Subgroup analysis was performed according to the status of EGFR, results of which indicated that the PFS of $\mathrm{ABCP}$ group $(\mathrm{n}=356)$ and $\mathrm{BCP}$ group $(\mathrm{n}=336)$ in EGFR wild-type (WT) population were 11.3 months and 6.8 months, respectively. Furthermore, 124/1202 EGFR-positive patients were randomized to three groups, including the ABCP $(n=34), A C P(n=45)$, or BCP $(n=45)$. The median OS was not estimated in the $\mathrm{ABCP}$, but it was 21.4 months in the $\mathrm{ACP}$ group and 18.7 months in BCP group (71). In addition, patients with advanced NSCLC receiving treatment with a combination of Nivolumab and Bevacizumab were recruited for a phase 1 study (NCT01454102), which aimed to evaluate whether the combination therapy improves PFS and OS. The experimental results indicate that the combined treatment group had significant safety, and the incidence of grade 3 and above adverse reactions is low. Therefore, it has shown excellent therapeutic effects compared to the single-agent treatment group (72). The combined treatment group had a median PFS of 37.1 weeks; however, in the Nivolumab monotherapy group, the median PFS of squamous cell carcinoma was 16 weeks, and the median PFS of non-squamous cell carcinoma was 21.4 weeks. Additionally, the median OS of the combined treatment group was 86.7 weeks, which is much larger than that of the monotherapy group (73). In 2019, results of the phase 1 study (NCT02443324) were reported. Among the total 27 enrolled patients with previously treated advanced NSCLC that received Ramucirumab plus Pembrolizumab, 8 patients achieved an objective response. Results also demonstrated that the disease control rate was $86 \%$, with a median PFS and OS of 9.7 and 26.2 months, respectively (74). Another phase 1 study (NCT02572687) indicated that the combination of Ramucirumab plus Durvalumab led to an enhancement of preliminary antitumor activity in heavy pretreated NSCLC patients with a median PFS of 1.7 months and OS of 12.4 months (75).

\section{Clinical Trials on RCC}

A first randomized phase 2 IMmotion150 study (NCT01984242) for patients with previously untreated mRCC treated with Atezolizumab combination Bevacizumab or single Atezolizumab or single sunitinib showed that the PFS of this combination group significantly improved within the population, whatever the PD-L1 status (76). Immunotherapy with PD-1 and PD-L1 inhibitors or combined with antiangiogenic therapy (i.e. VEGF inhibitors or CTLA-4 antibodies) has become a first line therapy for advanced RCC patients (77). Nivolumab plus Ipilimumab in a phase 3 trial (NCT02231749) has provided significant benefits in untreated intermediate and poor-risk RCC patients with a higher 18month OS rate of $75 \%$ and objective responses rate (ORR) of $42 \%$, compared to sunitinib with 18 -month OS rate of $60 \%$ and ORR of $27 \%$ (78). In 2018, an open-label, dose-finding and doseexpansion multicenter phase 1 study (NCT02493751) confirmed the disease control rate (DCR) of $78 \%$ with the security in the combination therapy of axitinib plus Avelumab in advanced RCC patients (79). Another phase 3 study (NCT02684006) validated that the combination of Avelumab plus axitinib enhanced the curative effect in patients with advanced RCC, leading to remarkable improvement in median PFS (13.8 months) and ORR (51.4\%), compared to treatment with sunitinib (8.4 months and 25.7\%, respectively) (80). In 2020, a pivotal phase 3 study (NCT02853331) demonstrated that Avelumab or Pembrolizumab Plus axitinib were more efficacious than sunitinib, a previous standard of care. This study recruited 861 metastatic renal cell carcinoma (mRCC) patients with results showing an improvement in PFS, a high response rate, and a low rate of intrinsic resistance (81). Recently, a phase 1b/2 study (NCT03136627) of Tivozanib combined with Nivolumab in patients with mRCC has been completed. The results demonstrated a promising antitumor efficacy with ORR of $56 \%$, DCR of $96 \%$ and median PFS of 18.9 months (82).

\section{Clinical Trials on HCC}

Phase 1 study (NCT02942329) of the VEGFR2 inhibitor apatinib plus anti-PD1 antibody SHR-1210 in patients with advanced hepatocellular carcinoma (HCC) has demonstrated manageable toxicity and encouraged clinical activity at recommended singleagent doses of both drugs (83). A phase $1 \mathrm{~b}$ clinical trial (NCT02715531) proved that combination of Bevacizumab and Atezolizumab profoundly improved the therapeutic effect 
TABLE 1 | Principal clinical trials for the approval of antiangiogenic and or immunotherapy agents.

\begin{tabular}{|c|c|c|c|c|c|c|c|c|}
\hline Drug & Indication & Phase & Pivotal study & PFS (Months) & OS (Months) & ORR & $\begin{array}{c}\text { First } \\
\text { posted }\end{array}$ & $\begin{array}{l}\text { Recruitment } \\
\text { status }\end{array}$ \\
\hline $\begin{array}{l}\text { Atezolizumab + } \\
\text { Bevacizumab }\end{array}$ & NSCLC & 3 & NCT02366143 & 8.3 vs 6.8 & 19.2 vs 14.7 & NA & 2015 & Completed \\
\hline Bevacizumab + Nivolumab & NSCLC & 1 & NCT01454102 & 9.3 vs 4.0 & 21.7 vs 14.1 & $8.0 \%$ vs $10.0 \%$ & 2011 & Completed \\
\hline $\begin{array}{l}\text { Ramucirumab + } \\
\text { Pembrolizumab }\end{array}$ & NSCLC & 1 & NCT02443324 & 9.7 & 26.2 & $30.0 \%$ & 2015 & $\begin{array}{l}\text { Active, not } \\
\text { recruiting }\end{array}$ \\
\hline Ramucirumab + Durvalumab & NSCLC & 1 & NCT02572687 & 2.7 & 11.0 & $11.0 \%$ & 2015 & Completed \\
\hline $\begin{array}{l}\text { Bevacizumab + } \\
\text { Atezolizumab }\end{array}$ & RCC & 2 & NCT01984242 & $\begin{array}{c}11.7 \text { vs } 8.4 \text { vs } \\
6.1\end{array}$ & NA & $\begin{array}{c}32.0 \% \text { vs } 29.0 \% \text { vs } \\
25.0 \%\end{array}$ & 2013 & Completed \\
\hline Nivolumab + Ipilimumab & $\mathrm{RCC}$ & 3 & NCT02231749 & 11.6 vs 8.4 & NA vs 26.0 & $9.0 \%$ vs $1.0 \%$ & 2014 & $\begin{array}{l}\text { Active, not } \\
\text { recruiting }\end{array}$ \\
\hline Axitinib + Avelumab & RCC & 1 & NCT02493751 & NA & NA & $27.0 \%$ vs $4.0 \%$ & 2015 & Completed \\
\hline Axitinib + Avelumab & $\mathrm{RCC}$ & 3 & NCT02684006 & 13.8 vs 8.4 & 11.6 vs 10.7 & $55.2 \%$ vs $25.5 \%$ & 2016 & $\begin{array}{l}\text { Active, not } \\
\text { recruiting }\end{array}$ \\
\hline Pembrolizumab + Axitinib & $\mathrm{RCC}$ & 3 & NCT02853331 & 17.1 vs. 11.1 & NA & $60.0 \%$ vs. $38.5 \%$ & 2016 & $\begin{array}{l}\text { Active, not } \\
\text { recruiting }\end{array}$ \\
\hline Tivozanib + Nivolumab & $\mathrm{RCC}$ & $1 / 2$ & NCT03136627 & $18.9 \%$ & NA & $56.0 \%$ & 2017 & Completed \\
\hline Apatinib + SHR-1210 & $\mathrm{HCC}$ & 1 & NCT02942329 & 2.9 & 11.4 & $30.8 \%$ & 2016 & Unknown \\
\hline $\begin{array}{l}\text { Bevacizumab + } \\
\text { Atezolizumab }\end{array}$ & $\mathrm{HCC}$ & 1 & NCT02715531 & 5.6 vs 3.4 & NA & $36.0 \%$ & 2016 & Completed \\
\hline Nivolumab + Ipilimumab & $\mathrm{HCC}$ & $1 / 2$ & NCT01658878 & NA & $\begin{array}{c}22.8 \text { vs } 12.5 \text { vs } \\
12.7\end{array}$ & $\begin{array}{c}32.0 \% \text { vs } 27.0 \% \text { vs } \\
29.0 \%\end{array}$ & 2012 & $\begin{array}{l}\text { Active, not } \\
\text { recruiting }\end{array}$ \\
\hline Lenvatinib + Pembrolizumab & $\mathrm{HCC}$ & 1 & NCT03006926 & 8.6 & 22.0 & $36.0 \%$ & 2016 & $\begin{array}{l}\text { Active, not } \\
\text { recruiting }\end{array}$ \\
\hline $\begin{array}{l}\text { Bevacizumab + } \\
\text { Atezolizumab }\end{array}$ & $\mathrm{HCC}$ & 3 & NCT03434379 & NA & NA & NA & 2018 & $\begin{array}{l}\text { Active, not } \\
\text { recruiting }\end{array}$ \\
\hline Bevacizumab + Dacarbazine & Melanoma & 2 & NCT01164007 & 5.5 & 11.4 & $18.9 \%$ & 2010 & Completed \\
\hline Bevacizumab + Ipilimumab & Melanoma & 1 & NCT00790010 & 9.0 & 25.1 & $19.6 \%$ & 2008 & $\begin{array}{l}\text { Active, not } \\
\text { recruiting }\end{array}$ \\
\hline Axitinib + Toripalimab & Melanoma & 1 & NCT03086174 & 7.5 & NA & $67.5 \%$ & 2017 & $\begin{array}{l}\text { Active, not } \\
\text { recruiting }\end{array}$ \\
\hline $\begin{array}{l}\text { Bevacizumab + } \\
\text { Atezolizumab }\end{array}$ & CRC & 2 & NCT0287319 & 4.4 vs 3.3 & NA & NA & Unknown & Unknown \\
\hline Bevacizumab + Nivolumab & $\mathrm{CRC}$ & 2 & NCT04072198 & NA & NA & NA & 2019 & Recruiting \\
\hline
\end{tabular}

NSCLC, non-small cell lung cancer; RCC, renal cell cancer; HCC, hepatocellular carcinoma; CRC, colorectal cancer; ORR, objective responses rate; RFS, progression-free survival; OS, overall survival. NA, Not available.

compared to the standard-of-care sorafenib in a phase 3 trial with ORR and DCR of $62 \%$ and $78 \%$, respectively. With regards to safety, the combination group demonstrated tolerable safety, with serious adverse events (AEs) rate of only $8 \%$ (84). CheckMate 040 is a phase $1 / 2$ randomized clinical trial (NCT01658878) which comprised of 148 HCC patients that were randomized 1:1:1 into three dosing arms (Nivolumab, alone or in combination with ipilimumab). These cohort results suggest that Nivolumab, plus Ipilimumab, may provide an improved ORR and OS, especially in arm A (lower dose Nivolumab and higher dose Ipilimumab), relative to anti-PD-L1 monotherapy (85). The combination of lenvatinib plus Pembrolizumab for unresectable HCC (uHCC) patients in the Phase $1 \mathrm{~b}$ trial (NCT03006926) represented a promising antitumor activity with an ORR of $46.0 \%$, and a median PFS of 9.3 months (86). Moreover, an ongoing double-blind randomized controlled phase 3 study (NCT03713593) of lenvatinib plus Pembrolizumab treatment of uHCC is currently being undertaken (77). Imbrave150 (NCT03434379), a randomized, multicenter phase 3 clinical study aims to evaluate the efficacy and safety of Atezolizumab plus Bevacizumab versus Sorafenib among patients with advanced HCC. The results indicated that, among 501 patients (336 in the combination group and 165 in the Sorafenib group) with HCC, the combination group showed a remarkable improvement in median PFS and OS with tolerated and controllable toxicity, compared to the Sorafenib group (87).

\section{Clinical Trials on Melanoma and CRC}

Dacarbazine in combination with Bevacizumab was studied in a phase 2 study (NCT01164007) of 40 unresectable/metastatic melanoma patients. The results from this study indicated that the treatment had an ORR of $18.9 \%$ and a median OS of 11.4 months, with no new toxicity (88). Preliminary results from the phase 1 clinical trial (NCT00790010) showed that Ipilimumab (CTLA-4 antibody) plus Bevacizumab (VEGF inhibitors) in patients with metastatic melanoma (MM) had favorable clinical outcomes, for reasons of increasing tumor vascular expression of ICAM-1 and VCAM-1 and lymphocyte infiltration in tumors (89). Another open-label phase $1 \mathrm{~b}$ trial (NCT03086174) validated the efficacy of axitinib in combination with Toripalimab among patients with advanced melanoma with an ORR of $48.3 \%$ and a median PFS of 7.5 months (90). In addition, a phase 2 study (NCT0287319) in 133 mCRC patients also demonstrated that the addition of Atezolizumab to Bevacizumab, as well as capecitabine, improved the median 
PFS of 4.4 months compared to that of 3.3 months in the modified intent-to-treat analysis (mITT) analysis group (91). Another open-label, multicenter phase 2 trial (NCT04072198) of FOLFOXIRI/Bevacizumab, in association with Nivolumab, was conducted in patients with mCRC. The results demonstrated that the combination was generally well tolerated, with an acceptable toxicity profile without any unexpected findings (92).

\section{FUTURE PROSPECTS}

Anti-tumor angiogenesis was found to be favorable to T-cell infiltration and drug delivery to the tumor, thereby enhancing the efficacy of immunotherapy. Additionally, immunotherapy can also increase tumor vascular normalization and form positive feedback to anti-angiogenesis. Therefore, the combination of anti-angiogenic agents and immunotherapy provides a new therapeutic approach for tumor patients. A large number of studies have demonstrated that the combination therapy has good clinical application prospects. However, the relationship between tumor angiogenesis and immune response is intricate, and some tough problems still need to be solved for future practical application.

Firstly, there is no way to identify tumor patients that can benefit from combination therapy (93), and anti-angiogenesis therapy has a lack of biomarkers, as mentioned above. In order to address the problem, oncologists have to identify the biomarkers that can be associated with patient groups that would be advantaged with this therapy. Secondly, the dose of each drug,

\section{REFERENCES}

1. Soria J-C, Mauguen A, Reck M, Sandler A, Saijo N, Johnson D, et al. Systematic Review and Meta-Analysis of Randomised, Phase Ii/lii Trials Adding Bevacizumab to Platinum-Based Chemotherapy as First-Line Treatment in Patients With Advanced Non-Small-Cell Lung Cancer. Ann Oncol (2012) 24(1):20-30. doi: 10.1093/annonc/mds590

2. Reck M, Kaiser R, Mellemgaard A, Douillard J, Orlov S, Krzakowski M, et al. Docetaxel Plus Nintedanib Versus Docetaxel Plus Placebo in Patients With Previously Treated Non-Small-Cell Lung Cancer (Lume-Lung 1): A Phase 3, Double-Blind, Randomised Controlled Trial. Lancet Oncol (2014) 15(2):14355. doi: 10.1016/s1470-2045(13)70586-2

3. Garon EB, Ciuleanu T-E, Arrieta O, Prabhash K, Syrigos KN, Goksel T, et al. Ramucirumab Plus Docetaxel Versus Placebo Plus Docetaxel for Second-Line Treatment of Stage Iv Non-Small-Cell Lung Cancer After Disease Progression on Platinum-Based Therapy (Revel): A Multicentre, Double-Blind, Randomised Phase 3 Trial. Lancet (2014) 384(9944):665-73. doi: 10.1016/ S0140-6736(14)60845-X

4. Pàez-Ribes M, Allen E, Hudock J, Takeda T, Okuyama H, Viñals F, et al. Antiangiogenic Therapy Elicits Malignant Progression of Tumors to Increased Local Invasion and Distant Metastasis. Cancer Cell (2009) 15 (3):220-31. doi: 10.1016/j.ccr.2009.01.027

5. Kindler HL, Niedzwiecki D, Hollis D, Sutherland S, Schrag D, Hurwitz H, et al. Gemcitabine Plus Bevacizumab Compared With Gemcitabine Plus Placebo in Patients With Advanced Pancreatic Cancer: Phase III Trial of the Cancer and Leukemia Group B (Calgb 80303). J Clin Oncol (2010) 28 (22):3617. doi: 10.1200/JCO.2010.28.1386

6. Keunen O, Johansson M, Oudin A, Sanzey M, Rahim SAA, Fack F, et al. AntiVegf Treatment Reduces Blood Supply and Increases Tumor Cell Invasion in the optimal sequence, and the time of the combination also remain significant. The high or low dose, simultaneous or sequential treatment, will have an effect on the efficacy of the combination therapy. Furthermore, studies have demonstrated that high doses of anti-angiogenic drugs can directly damage tumor blood vessels, which results in more serious disturbances of tumor microenvironment, such as hypoxia and immunosuppression (94). Therefore, it is necessary to choose the appropriate drug dosage, and optimize the schedule of tumor immunotherapy and anti-angiogenesis therapy in order to obtain improved anticancer efficacy. Moreover, the most frequent side effect of anti-angiogenic is hypertension (95). Therefore, primary or acquired resistance, including non-upregulation VEGF in tumors, changes in the TME, the presence of CSCs, and the patient with hypertension contribute to anti-angiogenesis failure (16). Besides, resistance to immunotherapy, including lack of tumor-infiltrating lymphocytes in the tumor, accumulating immunosuppressive cells in the TME and secreting immunosuppressive cytokines in the tumor cells, contributes significantly to failure of immunotherapy.

\section{AUTHOR CONTRIBUTIONS}

FL and $\mathrm{JH}$ contributed to the study design. $\mathrm{HH}$ and $\mathrm{YC}$ were responsible for data collection. ST, YH, and SF drafted and prepared the manuscript. SW worked for the table and figures. All authors participated in the data interpretation and contributed to the manuscript writing with important intellectual input. All authors approved the final version of the manuscript.

Glioblastoma. Proc Natl Acad Sci (2011) 108(9):3749-54. doi: 10.1073/ pnas. 1014480108

7. Ebos JM, Lee CR, Cruz-Munoz W, Bjarnason GA, Christensen JG, Kerbel RS. Accelerated Metastasis After Short-Term Treatment With a Potent Inhibitor of Tumor Angiogenesis. Cancer Cell (2009) 15(3):232-9. doi: 10.1016/ j.ccr.2009.01.021

8. Ramadan W, Zaher D, Altaie A, Talaat I, Elmoselhi A. Potential Therapeutic Strategies for Lung and Breast Cancers Through Understanding the AntiAngiogenesis Resistance Mechanisms. Int J Mol Sci (2020) 21(2):565. doi: $10.3390 /$ ijms 21020565

9. Missiaen R, Mazzone M, Bergers G. The Reciprocal Function and Regulation of Tumor Vessels and Immune Cells Offers New Therapeutic Opportunities in Cancer. Semin Cancer Biol (2018) 52:107-16. doi: 10.1016/ j.semcancer.2018.06.002

10. Craven K, Gore J, Korc M. Overview of Pre-Clinical and Clinical Studies Targeting Angiogenesis in Pancreatic Ductal Adenocarcinoma. Cancer Lett (2016) 381(1):201-10. doi: 10.1016/j.canlet.2015.11.047

11. Ioannidou E, Moschetta M, Shah S, Parker J, Ozturk M, Pappas-Gogos G, et al. Angiogenesis and Anti-Angiogenic Treatment in Prostate Cancer: Mechanisms of Action and Molecular Targets. Int J Mol Sci (2021) 22 (18):9926. doi: 10.3390/ijms22189926

12. Kerbel R. Reappraising Antiangiogenic Therapy for Breast Cancer. Breast (Edinburgh Scotland) (2011) 20 Suppl 3(0 3):S56-60. doi: 10.1016/s0960-9776 (11)70295-8

13. Ahir B, Engelhard H, Lakka S. Tumor Development and Angiogenesis in Adult Brain Tumor: Glioblastoma. Mol Neurobiol (2020) 57(5):2461-78. doi: 10.1007/s12035-020-01892-8

14. Cascone T, Herynk M, Xu L, Du Z, Kadara H, Nilsson M, et al. Upregulated Stromal Egfr and Vascular Remodeling in Mouse Xenograft Models of 
Angiogenesis Inhibitor-Resistant Human Lung Adenocarcinoma. J Clin Invest (2011) 121(4):1313-28. doi: 10.1172/jci42405

15. Huijbers E, van Beijnum J, Thijssen V, Sabrkhany S, Nowak-Sliwinska P, Griffioen A. Role of the Tumor Stroma in Resistance to Anti-Angiogenic Therapy. Drug Resist Updat: Rev Comment Antimicrob Anticancer Chemother (2016) 25:26-37. doi: 10.1016/j.drup.2016.02.002

16. Chandra A, Rick J, Yagnik G, Aghi MK. Autophagy as a Mechanism for AntiAngiogenic Therapy Resistance. Semin Cancer Biol (2020) 66:75-88. doi: 10.1016/j.semcancer.2019.08.031

17. Li C, Teixeira A, Zhu H, Ten Dijke P. Cancer Associated-Fibroblast-Derived Exosomes in Cancer Progression. Mol Cancer (2021) 20(1):154. doi: 10.1186/ s12943-021-01463-y

18. Mao X, Xu J, Wang W, Liang C, Hua J, Liu J, et al. Crosstalk Between CancerAssociated Fibroblasts and Immune Cells in the Tumor Microenvironment: New Findings and Future Perspectives. Mol Cancer (2021) 20(1):131. doi: 10.1186/s12943-021-01428-1

19. Deepak K, Vempati R, Nagaraju G, Dasari V, Nagini S, Rao D, et al. S NTumor Microenvironment: Challenges and Opportunities in Targeting Metastasis of Triple Negative Breast Cancer. Pharmacol Res (2020) 153:104683. doi: $10.1016 /$ j.phrs.2020.104683

20. Zhang H, Wang Z, Peng Q, Liu Y-Y, Zhang W, Wu L, et al. Tumor Refractoriness to Endostatin Anti-Angiogenesis Is Associated With the Recruitment of Cd11b+ Gr1+ Myeloid Cells and Inflammatory Cytokines. Tumori J (2013) 99(6):723-33. doi: 10.1700/1390.15462

21. Wang Z, Li Z, Wang Y, Cao D, Wang X, Jiang M, et al. Versican Silencing Improves the Antitumor Efficacy of Endostatin by Alleviating Its Induced Inflammatory and Immunosuppressive Changes in the Tumor Microenvironment. Oncol Rep (2015) 33(6):2981-91. doi: 10.3892/or. 2015.3903

22. Wang L, Wang J, Li Z, Liu Y, Jiang M, Li Y, et al. Silencing Stem Cell Factor Attenuates Stemness and Inhibits Migration of Cancer Stem Cells Derived From Lewis Lung Carcinoma Cells. Tumor Biol (2016) 37(6):7213-27. doi: 10.1007/s13277-015-4577-6

23. Wang L, Guo H, Lin C, Yang L, Wang X. Enrichment and Characterization of Cancer Stem-Like Cells From a Cervical Cancer Cell Line. Mol Med Rep (2014) 9(6):2117-23. doi: 10.3892/mmr.2014.2063

24. Lin C, Wang L, Wang H, Yang L, Guo H, Wang X. Tanshinone Iia Inhibits Breast Cancer Stem Cells Growth In Vitro and In Vivo Through Attenuation of Il-6/Stat3/Nf-Kb Signaling Pathways. J Cell Biochem (2013) 114(9):206170. doi: $10.1002 /$ jcb. 24553

25. Cao L, Fan X, Jing W, Liang Y, Chen R, Liu Y, et al. Osteopontin Promotes a Cancer Stem Cell-Like Phenotype in Hepatocellular Carcinoma Cells Via an Integrin-Nf-Kb-Hif-1 $\alpha$ Pathway. Oncotarget (2015) 6(9):6627. doi: 10.18632/ oncotarget. 3113

26. Santoyo-Ramos P, Likhatcheva M, García-Zepeda EA, Castañeda-Patlán MC, Robles-Flores M. Hypoxia-Inducible Factors Modulate the Stemness and Malignancy of Colon Cancer Cells by Playing Opposite Roles in Canonical Wnt Signaling. PloS One (2014) 9(11):e112580. doi: 10.1371/journal.pone. 0112580

27. Zhang L, Huang R, Shen Y, Liu J, Wu Y, Jin J, et al. Viaenhanced Anti-Tumor Efficacy by Inhibiting Hif- $1 \alpha$ to Reprogram Tams Core-Satellite Upconverting Nanoparticles With Curcumin Mediated Photodynamic Therapy. Biomater Sci (2021) 9:6403-15. doi: 10.1039/d1bm00675d

28. Zahavi D, Weiner L. Tumor Mechanisms of Resistance to Immune Attack. Prog Mol Biol Trans Sci (2019) 164:61-100. doi: 10.1016/bs.pmbts.2019.03.009

29. Whiteside T. Immune Suppression in Cancer: Effects on Immune Cells, Mechanisms and Future Therapeutic Intervention. Semin Cancer Biol (2006) 16(1):3-15. doi: 10.1016/j.semcancer.2005.07.008

30. Finke JH, Rini B, Ireland J, Rayman P, Richmond A, Golshayan A, et al. Sunitinib Reverses Type-1 Immune Suppression and Decreases T-Regulatory Cells in Renal Cell Carcinoma Patients. Clin Cancer Res (2008) 14(20):667482. doi: 10.1158/1078-0432.CCR-07-5212

31. Noguera-Troise I, Daly C, Papadopoulos NJ, Coetzee S, Boland P, Gale NW, et al. Blockade of Dll4 Inhibits Tumour Growth by Promoting NonProductive Angiogenesis. Nature (2006) 444(7122):1032. doi: 10.1038/ nature 05355

32. Welford AF, Biziato D, Coffelt SB, Nucera S, Fisher M, Pucci F, et al. Tie2Expressing Macrophages Limit the Therapeutic Efficacy of the Vascular-
Disrupting Agent Combretastatin A4 Phosphate in Mice. J Clin Invest (2011) 121(5):1969-73. doi: 10.1172/JCI44562

33. Huang J, Soffer SZ, Kim ES, McCrudden KW, Huang J, New T, et al. Vascular Remodeling Marks Tumors That Recur During Chronic Suppression of Angiogenesis11nih U10 Ca13539-27, Subcontract 6641 (Jk), Nih 1 R01 Ca08895101-A1 (Dy), Pediatric Cancer Foundation, and Sorkin Gift Fund. Note: J. Huang and Sz Soffer Contributed Equally to This Work. Mol Cancer Res (2004) 2(1):36-42.

34. Wu X, Giobbie-Hurder A, Liao X, Connelly C, Connolly E, Li J, et al. Angiopoietin-2 as a Biomarker and Target for Immune Checkpoint Therapy. Cancer Immunol Res (2017) 5(1):17-28. doi: 10.1158/23266066.Cir-16-0206

35. Schmittnaegel M, Rigamonti N, Kadioglu E, Cassará A, Wyser Rmili C, Kiialainen A, et al. Dual Angiopoietin-2 and Vegfa Inhibition Elicits Antitumor Immunity That Is Enhanced by Pd-1 Checkpoint Blockade. Sci Trans Med (2017) 9(385):eaak9670. doi: 10.1126/scitranslmed.aak9670

36. Tobias J, Steinberger P, Drinić M, Wiedermann U. Emerging Targets for Anticancer Vaccination: Pd-1. ESMO Open (2021) 6(5):100278. doi: 10.1016/ j.esmoop.2021.100278

37. Shin C, Kim S, Jo Y. Extending Traditional Antibody Therapies: Novel Discoveries in Immunotherapy and Clinical Applications. Mol Ther Oncolytics (2021) 22:166-79. doi: 10.1016/j.omto.2021.08.005

38. Ohm J, Carbone D. Vegf as a Mediator of Tumor-Associated Immunodeficiency. Immunol Res (2001) 23:263-72. doi: 10.1385/ir:23:2-3:263

39. Garber K. Promising Early Results for Immunotherapy-Antiangiogenesis Combination. J Natl Cancer Inst (2014) 106(11):dju392. doi: 10.1093/jnci/ dju392

40. Woo S, Turnis M, Goldberg M, Bankoti J, Selby M, Nirschl C, et al. Immune Inhibitory Molecules Lag-3 and Pd-1 Synergistically Regulate T-Cell Function to Promote Tumoral Immune Escape. Cancer Res (2012) 72(4):917-27. doi: 10.1158/0008-5472.Can-11-1620

41. De Palma M, Biziato D, Petrova TV. Microenvironmental Regulation of Tumour Angiogenesis. Nat Rev Cancer (2017) 17(8):457-74. doi: 10.1038/ nrc.2017.51

42. Wallin J, Bendell J, Funke R, Sznol M, Korski K, Jones S, et al. Atezolizumab in Combination With Bevacizumab Enhances Antigen-Specific T-Cell Migration in Metastatic Renal Cell Carcinoma. Nat Commun (2016) 7:12624. doi: $10.1038 /$ ncomms12624

43. Huang Y, Goel S, Duda D, Fukumura D, Jain R. Vascular Normalization as an Emerging Strategy to Enhance Cancer Immunotherapy. Cancer Res (2013) 73 (10):2943-8. doi: 10.1158/0008-5472.Can-12-4354

44. Hinshaw D, Shevde L. The Tumor Microenvironment Innately Modulates Cancer Progression. Cancer Res (2019) 79(18):4557-66. doi: 10.1158/00085472.Can-18-3962

45. Khan K, Kerbel R. Improving Immunotherapy Outcomes With AntiAngiogenic Treatments and Vice Versa. Nat Rev Clin Oncol (2018) 15 (5):310-24. doi: 10.1038/nrclinonc.2018.9

46. Soiffer R, Hodi F, Haluska F, Jung K, Gillessen S, Singer S, et al. Vaccination With Irradiated, Autologous Melanoma Cells Engineered to Secrete Granulocyte-Macrophage Colony-Stimulating Factor by AdenoviralMediated Gene Transfer Augments Antitumor Immunity in Patients With Metastatic Melanoma. J Clin Oncol: Off J Am Soc Clin Oncol (2003) 21 (17):3343-50. doi: 10.1200/jco.2003.07.005

47. Dranoff G, Jaffee E, Lazenby A, Golumbek P, Levitsky H, Brose K, et al. Vaccination With Irradiated Tumor Cells Engineered to Secrete Murine Granulocyte-Macrophage Colony-Stimulating Factor Stimulates Potent, Specific, and Long-Lasting Anti-Tumor Immunity. Proc Natl Acad Sci USA (1993) 90(8):3539-43. doi: 10.1073/pnas.90.8.3539

48. Jaffee E, Hruban R, Biedrzycki B, Laheru D, Schepers K, Sauter P, et al. Novel Allogeneic Granulocyte-Macrophage Colony-Stimulating Factor-Secreting Tumor Vaccine for Pancreatic Cancer: A Phase I Trial of Safety and Immune Activation. J Clin Oncol: Off J Am Soc Clin Oncol (2001) 19 (1):145-56. doi: 10.1200/jco.2001.19.1.145

49. Nemunaitis J. Gvax (Gmcsf Gene Modified Tumor Vaccine) in Advanced Stage Non Small Cell Lung Cancer. J Control Release: Off J Control Release Soc (2003) 91:225-31. doi: 10.1016/s0168-3659(03)00210-4

50. Salgia R, Lynch T, Skarin A, Lucca J, Lynch C, Jung K, et al. Vaccination With Irradiated Autologous Tumor Cells Engineered to Secrete Granulocyte- 
Macrophage Colony-Stimulating Factor Augments Antitumor Immunity in Some Patients With Metastatic Non-Small-Cell Lung Carcinoma. J Clin Oncol: Off J Am Soc Clin Oncol (2003) 21(4):624-30. doi: 10.1200/jco. 2003.03.091

51. Dikov M, Ohm J, Ray N, Tchekneva E, Burlison J, Moghanaki D, et al. Differential Roles of Vascular Endothelial Growth Factor Receptors 1 and 2 in Dendritic Cell Differentiation. J Immunol (Baltimore Md: 1950) (2005) 174 (1):215-22. doi: 10.4049/jimmunol.174.1.215

52. Séguier S, Tartour E, Guérin C, Couty L, Lemitre M, Lallement L, et al. Inhibition of the Differentiation of Monocyte-Derived Dendritic Cells by Human Gingival Fibroblasts. PloS One (2013) 8(8):e70937. doi: 10.1371/ journal.pone.0070937

53. Gabrilovich D, Ishida T, Oyama T, Ran S, Kravtsov V, Nadaf S, et al. Vascular Endothelial Growth Factor Inhibits the Development of Dendritic Cells and Dramatically Affects the Differentiation of Multiple Hematopoietic Lineages in Vivo: Presented in Part at the Keystone Symposium "Cellular and Molecular Biology of Dendritic Cells", Santa Fe, Nm, March 3-9, 1998, and at the Annual Meeting of the American Association for Cancer Research, March 28-April 1, 1998. Blood (1998) 92(11):4150-66. doi: 10.1182/blood. v92.11.4150

54. Gabrilovich DI, Chen HL, Girgis KR, Cunningham HT, Meny GM, Nadaf S, et al. Production of Vascular Endothelial Growth Factor by Human Tumors Inhibits the Functional Maturation of Dendritic Cells. Nat Med (1996) 2 (10):1096. doi: 10.1038/nm1096-1096

55. Ohm JE, Gabrilovich DI, Sempowski GD, Kisseleva E, Parman KS, Nadaf S, et al. Vegf Inhibits T-Cell Development and May Contribute to TumorInduced Immune Suppression. Blood (2003) 101(12):4878-86. doi: 10.1182/ blood-2002-07-1956

56. Shi S, Wang R, Chen Y, Song H, Chen L, Huang G. Combining Antiangiogenic Therapy With Adoptive Cell Immunotherapy Exerts Better Antitumor Effects in Non-Small Cell Lung Cancer Models. PloS One (2013) 8 (6):e65757. doi: 10.1371/journal.pone.0065757

57. Meder L, Schuldt P, Thelen M, Schmitt A, Dietlein F, Klein S, et al. Combined Vegf and Pd-L1 Blockade Displays Synergistic Treatment Effects in an Autochthonous Mouse Model of Small Cell Lung Cancer. Cancer Res (2018) 78(15):4270-81. doi: 10.1158/0008-5472.Can-17-2176

58. Yasuda S, Sho M, Yamato I, Yoshiji H, Wakatsuki K, Nishiwada S, et al. Simultaneous Blockade of Programmed Death 1 and Vascular Endothelial Growth Factor Receptor 2 (Vegfr2) Induces Synergistic Anti-Tumour Effect in Vivo. Clin Exp Immunol (2013) 172(3):500-6. doi: 10.1111/cei.12069

59. Gérard A, Cope A, Kemper C, Alon R, Köchl R. Lfa-1 in T Cell Priming, Differentiation, and Effector Functions. Trends Immunol (2021) 42(8):706-22. doi: 10.1016/j.it.2021.06.004

60. Bouzin C, Brouet A, De Vriese J, DeWever J, Feron O. Effects of Vascular Endothelial Growth Factor on the Lymphocyte-Endothelium Interactions: Identification of Caveolin-1 and Nitric Oxide as Control Points of Endothelial Cell Anergy. J Immunol (2007) 178(3):1505-11. doi: 10.4049/jimmunol. 178.3.1505

61. Motz GT, Santoro SP, Wang L-P, Garrabrant T, Lastra RR, Hagemann IS, et al. Tumor Endothelium Fasl Establishes a Selective Immune Barrier Promoting Tolerance in Tumors. Nat Med (2014) 20(6):607. doi: 10.1038/ nm.3541

62. Ko JS, Zea AH, Rini BI, Ireland JL, Elson P, Cohen P, et al. Sunitinib Mediates Reversal of Myeloid-Derived Suppressor Cell Accumulation in Renal Cell Carcinoma Patients. Clin Cancer Res (2009) 15(6):2148-57. doi: 10.1158/ 1078-0432.CCR-08-1332

63. Voron T, Colussi O, Marcheteau E, Pernot S, Nizard M, Pointet A-L, et al. Vegf-A Modulates Expression of Inhibitory Checkpoints on Cd8+ T Cells in Tumors. J Exp Med (2015) 212(2):139-48. doi: 10.1084/jem.20140559

64. Terme M, Pernot S, Marcheteau E, Sandoval F, Benhamouda N, Colussi O, et al. Vegfa-Vegfr Pathway Blockade Inhibits Tumor-Induced Regulatory TCell Proliferation in Colorectal Cancer. Cancer Res (2013) 73(2):539-49. doi: 10.1158/0008-5472.CAN-12-2325

65. Kwilas AR, Ardiani A, Donahue RN, Aftab DT, Hodge JW. Dual Effects of a Targeted Small-Molecule Inhibitor (Cabozantinib) on Immune-Mediated Killing of Tumor Cells and Immune Tumor Microenvironment Permissiveness When Combined With a Cancer Vaccine. J Trans Med (2014) 12(1):294. doi: 10.1186/s12967-014-0294-y
66. Li B, Lalani A, Harding T, Luan B, Koprivnikar K, Huan Tu G, et al. Vascular Endothelial Growth Factor Blockade Reduces Intratumoral Regulatory T Cells and Enhances the Efficacy of a Gm-Csf-Secreting Cancer Immunotherapy. Clin Cancer Res: An Off J Am Assoc Cancer Res (2006) 12(22):6808-16. doi: 10.1158/1078-0432.Ccr-06-1558

67. Holash J, Davis S, Papadopoulos N, Croll S, Ho L, Russell M, et al. Vegf-Trap: A Vegf Blocker With Potent Antitumor Effects. Proc Natl Acad Sci USA (2002) 99(17):11393-8. doi: 10.1073/pnas.172398299

68. Mortara L, Benest A, Bates D, Noonan D. Can the Co-Dependence of the Immune System and Angiogenesis Facilitate Pharmacological Targeting of Tumours? Curr Opin Pharmacol (2017) 35:66-74. doi: 10.1016/j.coph.2017.05.009

69. Wu J, Zhao X, Sun Q, Jiang Y, Zhang W, Luo J, et al. Synergic Effect of Pd-1 Blockade and Endostar on the Pi3k/Akt/Mtor-Mediated Autophagy and Angiogenesis in Lewis Lung Carcinoma Mouse Model. Biomed Pharmacother $=$ Biomed Pharmacother (2020) 125:109746. doi: 10.1016/ j.biopha.2019.109746

70. Socinski MA, Jotte RM, Cappuzzo F, Orlandi F, Stroyakovskiy D, Nogami N, et al. Atezolizumab for First-Line Treatment of Metastatic Nonsquamous Nsclc. N Engl J Med (2018) 378(24):2288-301. doi: 10.1056/ NEJMoa1716948

71. Reck M, Mok TSK, Nishio M, Jotte RM, Cappuzzo F, Orlandi F, et al. Atezolizumab Plus Bevacizumab and Chemotherapy in Non-Small-Cell Lung Cancer (Impower150): Key Subgroup Analyses of Patients With Egfr Mutations or Baseline Liver Metastases in a Randomised, Open-Label Phase 3 Trial. Lancet Respir Med (2019) 7(5):387-401. doi: 10.1016/s2213-2600(19) 30084-0

72. Hellmann M, Rizvi N, Goldman J, Gettinger S, Borghaei H, Brahmer J, et al. Nivolumab Plus Ipilimumab as First-Line Treatment for Advanced NonSmall-Cell Lung Cancer (Checkmate 012): Results of an Open-Label, Phase 1, Multicohort Study. Lancet Oncol (2017) 18(1):31-41. doi: 10.1016/s1470-2045 (16)30624-6

73. Rizvi N, Antonia S. F Shepherd. Nivolumab (Anti-Pd-1; Bms-936558, Ono-4538) Maintenance as Monotherapy or in Combination With Bevacizumab (Bev) for Non-Small Cell Lung Cancer (Nsclc) Previously Treated With Chemotherapy: Metastatic Non-Small Cell Lung Cancer. Int $J$ Radiat Oncol• Biol• Phys (2014) 90(5):S32. doi: 10.1016/j.ijrobp.2014.08.206

74. Herbst RS, Arkenau H-T, Santana-Davila R, Calvo E, Paz-Ares L, Cassier PA, et al. Ramucirumab Plus Pembrolizumab in Patients With Previously Treated Advanced Non-Small-Cell Lung Cancer, Gastro-Oesophageal Cancer, or Urothelial Carcinomas (Jvdf): A Multicohort, Non-Randomised, OpenLabel, Phase 1a/B Trial. Lancet Oncol (2019) 20(8):1109-23. doi: 10.1016/ s1470-2045(19)30458-9

75. Bang YJ, Golan T, Dahan L, Fu S, Moreno V, Park K, et al. Ramucirumab and Durvalumab for Previously Treated, Advanced Non-Small-Cell Lung Cancer, Gastric/Gastro-Oesophageal Junction Adenocarcinoma, or Hepatocellular Carcinoma: An Open-Label, Phase Ia/B Study (Jvdj). Eur J Cancer (2020) 137:272-84. doi: 10.1016/j.ejca.2020.06.007

76. McDermott DF, Huseni MA, Atkins MB, Motzer RJ, Rini BI, Escudier B, et al. Clinical Activity and Molecular Correlates of Response to Atezolizumab Alone or in Combination With Bevacizumab Versus Sunitinib in Renal Cell Carcinoma. Nat Med (2018) 24(6):749-57. doi: 10.1038/s41591-018-0053-3

77. Chambers A, Kundranda M, Rao S, Mahmoud F, Niu J. Anti-Angiogenesis Revisited: Combination With Immunotherapy in Solid Tumors. Curr Oncol Rep (2021) 23(9):100. doi: 10.1007/s11912-021-01099-7

78. Motzer RJ, Tannir NM, McDermott DF, Aren Frontera O, Melichar B, Choueiri TK, et al. Nivolumab Plus Ipilimumab Versus Sunitinib in Advanced Renal-Cell Carcinoma. N Engl J Med (2018) 378(14):1277-90. doi: 10.1056/NEJMoa1712126

79. Choueiri TK, Larkin J, Oya M, Thistlethwaite F, Martignoni M, Nathan P, et al. Preliminary Results for Avelumab Plus Axitinib as First-Line Therapy in Patients With Advanced Clear-Cell Renal-Cell Carcinoma (Javelin Renal 100): An Open-Label, Dose-Finding and Dose-Expansion, Phase 1b Trial. Lancet Oncol (2018) 19(4):451-60. doi: 10.1016/s1470-2045(18)30107-4

80. Motzer RJ, Penkov K, Haanen J, Rini B, Albiges L, Campbell MT, et al. Avelumab Plus Axitinib Versus Sunitinib for Advanced Renal-Cell Carcinoma. N Engl J Med (2019) 380(12):1103-15. doi: 10.1056/NEJMoa1816047

81. Grimm MO, Leucht K, Grunwald V, Foller S. New First Line Treatment Options of Clear Cell Renal Cell Cancer Patients With Pd-1 or Pd-L1 
Immune-Checkpoint Inhibitor-Based Combination Therapies. J Clin Med (2020) 9(2):565. doi: 10.3390/jcm9020565

82. Albiges L, Barthelemy P, Gross-Goupil M, Negrier S, Needle MN, Escudier B. Tinivo: Safety and Efficacy of Tivozanib-Nivolumab Combination Therapy in Patients With Metastatic Renal Cell Carcinoma. Ann Oncol (2021) 32(1):97102. doi: $10.1016 /$ j.annonc.2020.09.021

83. Xu J, Zhang Y, Jia R, Yue C, Chang L, Liu R, et al. Anti-Pd-1 Antibody Shr-1210 Combined With Apatinib for Advanced Hepatocellular Carcinoma, Gastric, or Esophagogastric Junction Cancer: An Open-Label, Dose Escalation and Expansion Study. Clin Cancer Res (2019) 25(2):515-23. doi: 10.1158/1078-0432.CCR-18-2484

84. Lee MS, Ryoo B-Y, Hsu C-H, Numata K, Stein S, Verret W, et al. Atezolizumab With or Without Bevacizumab in Unresectable Hepatocellular Carcinoma (Go30140): An Open-Label, Multicentre, Phase 1b Study. Lancet Oncol (2020) 21(6):808-20. doi: 10.1016/s1470-2045(20)30156-x

85. Yau T, Kang YK, Kim TY, El-Khoueiry AB, Santoro A, Sangro B, et al. Efficacy and Safety of Nivolumab Plus Ipilimumab in Patients With Advanced Hepatocellular Carcinoma Previously Treated With Sorafenib: The Checkmate 040 Randomized Clinical Trial. JAMA Oncol (2020) 6(11): e204564. doi: 10.1001/jamaoncol.2020.4564

86. Finn RS, Ikeda M, Zhu AX, Sung MW, Baron AD. Phase Ib Study of Lenvatinib Plus Pembrolizumab in Patients With Unresectable Hepatocellular Carcinoma. J Clin Oncol (2020) 38(26):2960-70. doi: $10.1200 / J C O .20 .00808$

87. Galle PR, Finn RS, Qin S, Ikeda M, Zhu AX, Kim T-Y, et al. Patient-Reported Outcomes With Atezolizumab Plus Bevacizumab Versus Sorafenib in Patients With Unresectable Hepatocellular Carcinoma (Imbrave150): An Open-Label, Randomised, Phase 3 Trial. Lancet Oncol (2021) 22(7):991-1001. doi: 10.1016/s1470-2045(21)00151-0

88. Ferrucci PF, Minchella I, Mosconi M, Gandini S, Verrecchia F, Cocorocchio E, et al. Dacarbazine in Combination With Bevacizumab for the Treatment of Unresectable/Metastatic Melanoma: A Phase II Study. Melanoma Res (2015) 25(3):239-45. doi: 10.1097/CMR.0000000000000146

89. Wu X, Giobbie-Hurder A, Liao X, Lawrence D, McDermott D, Zhou J, et al. Vegf Neutralization Plus Ctla-4 Blockade Alters Soluble and Cellular Factors Associated With Enhancing Lymphocyte Infiltration and Humoral Recognition in Melanoma. Cancer Immunol Res (2016) 4(10):858-68. doi: 10.1158/2326-6066.CIR-16-0084

90. Sheng X, Yan X, Chi Z, Si L, Cui C, Tang B. Axitinib in Combination With Toripalimab, a Humanized Immunoglobulin G 4 Monoclonal Antibody
Against Programmed Cell Death-1, in Patients With Metastatic Mucosal Melanoma: An Open-Label Phase Ib Trial. J Clin Oncol (2019) 37(32):298799. doi: $10.1200 / J C O .19$

91. Mettu NB, Twohy E, Ou F-S, Halfdanarson TR, Lenz HJ, Breakstone R. Bacci: A Phase Ii Randomized, Double-Blind, Multicenter, Placebocontrolled Study of Capecitabine (C) Bevacizumab (B) Plus Atezolizumab (a) or Placebo (P) in Refractory Metastatic Colorectal Cancer (Mcrc): An Accru Network Study. Ann Oncol (2019) 30(Supplement 5):203. doi: 10.1093/annonc/mdz246.011

92. Damato A, Berselli A, Iachetta F, Romagnani A, Larocca M, Arias AG. Preliminary Safety Analysis of Phase Ii Open-Label Nivacor Trial (Goirc03-2018) in Patients With Advanced Colorectal Cancer Ras or Braf Mutated. J Clin Oncol (2021) 39:37. doi: 10.1200/jco.2021.39.3_suppl.37

93. Ramjiawan RR, Griffioen AW, Duda DG. Anti-Angiogenesis for Cancer Revisited: Is There a Role for Combinations With Immunotherapy? Angiogenesis (2017) 20(2):185-204. doi: 10.1007/s10456-017-9552-y

94. Jain RK. Antiangiogenesis Strategies Revisited: From Starving Tumors to Alleviating Hypoxia. Cancer Cell (2014) 26(5):605-22. doi: 10.1016/ j.ccell.2014.10.006

95. des Guetz G, Uzzan B, Chouahnia K, Morère J. Cardiovascular Toxicity of Anti-Angiogenic Drugs. Target Oncol (2011) 6(4):197-202. doi: 10.1007/ s11523-011-0204-7

Conflict of Interest: The authors declare that the research was conducted in the absence of any commercial or financial relationships that could be construed as a potential conflict of interest.

Publisher's Note: All claims expressed in this article are solely those of the authors and do not necessarily represent those of their affiliated organizations, or those of the publisher, the editors and the reviewers. Any product that may be evaluated in this article, or claim that may be made by its manufacturer, is not guaranteed or endorsed by the publisher.

Copyright $\odot 2022 \mathrm{Hu}$, Chen, Tan, Wu, Huang, Fu, Luo and He. This is an openaccess article distributed under the terms of the Creative Commons Attribution License (CC BY). The use, distribution or reproduction in other forums is permitted, provided the original author(s) and the copyright owner(s) are credited and that the original publication in this journal is cited, in accordance with accepted academic practice. No use, distribution or reproduction is permitted which does not comply with these terms. 